\title{
Revealing the atomic-level organization of a bacterial microinjection nanodevice
}

Alok K. Mitra ${ }^{1}$, Ambroise Desfosses ${ }^{1}$, Hariprasad Venugopal ${ }^{1}$, Tapan Joshi ${ }^{1}$, Jae Hyun ${ }^{2}$, Pushpanjali Bhardwaj ${ }^{3}$, Mark Hurst ${ }^{3}$

${ }^{1}$ School Of Biological Sciences, University Of Auckland, Auckland, New Zealand, ${ }^{2}$ Korea Basic Science Institute, Daejeon, Korea, Rep., ${ }^{3}$ AgResearch, Lincoln Research Centre, Christchurch, New Zealand

E-mail: a.mitra@auckland.ac.nz

Nature has evolved sophisticated molecular machinery for specific biology. One such example is the multi-protein secretion systems (type I-type VII) that bacteria utilize to transfer effector molecules to confer survival advantage that can lead to pathogenicity towards other bacteria or eukaryotic hosts. These include microinjection systems- an impressive example of evolutionary engineering that can form templates for design of contemporary nano-devices. We have focussed on the antifeeding prophage (Afp)1, a plasmid-encoded mobile protein microinjection device produced by the bacteria Serratia entomophila that shares common features with bacteriophage T4 tail and type VI secretion systems (T6SS). Afp delivers a protein toxin to kill larvae of Costelytra zealandica, an insect pest endemic to New Zealand. Due to the fact that unlike for the other related systems Afp has a eukaryotic host, this has raised the possibility that apart from the benefit of using it as a chemical-free biological pesticide, Afp may be tailored for targeted delivery such as in anti-tumour immunotherapy. Afp, a bullet-shaped $\sim 110 \mathrm{~nm}$ long assembly, is composed of no less than 18 different proteins that are distributed in 3 distinct regions, the base plate with a cell puncturing device, the contractile polymeric sheath covering an inner tube, and a tapered end distal from the baseplate2,3. Afp elaborates two different configurations, the resting and the functional state wherein the sheath is contracted, the inner tube is partially exposed and the toxin is extruded into the host. We have recorded high-resolution cryo-EM (electron microscopy) images of both states of Afp and have solved the two structures at $\sim 3 \AA$ resolution. This analysis combined single-particle cryo-EM with helical reconstruction that is appropriate to the different parts of the macromolecular complex. The high-resolution achieved allowed for de novo atomic modeling of the constituent proteins and the positioning of the various proteins delineating the full organization of the assembly. As revealed by the 3D structures, an insight into the dynamics of the macromolecular assembly will be discussed; in particular, the transition from the resting to the contracted has been visualized in terms of the alteration in the packing and rearrangement of the sheath proteins(s) at atomic level.

[1] Hurst, M. R., Glare, T. R., Jackson, T. A. (2004). J Bacteriol. 186:5116-28.

[2] Heymann, J. B., Bartho J. D., Rybakova, D., Venugopal, H. P., Winkler, D.C., Sen, A., Hurst, M. R., Mitra, A. K. (2013). J Biol Chem. 288:25276-84.

[3] Rybakova, D., Radjainia, M., Turner, A., Sen, A., Mitra, A. K., Hurst, M. R. (2013). Mol Microbiol. 89:702-14.

Keywords: tailocin, cryo-EM, single particle analysis 